\title{
A LOWER BOUND OF MAXIMAL DILATATIONS OF QUASICONFORMAL AUTOMORPHISMS ACTING ON FUCHSIAN GROUPS
}

\author{
EGE FUJIKAWA \\ To the memory of Professor Nobuyuki Suita
}

\section{INTRODUCTION}

Let $\mathbb{H}$ be the upper half-plane equipped with the hyperbolic metric $|d z| / \operatorname{Im} z$, and $\Gamma$ a Fuchsian group acting on $\mathbb{H}$. A subset $S \subset \mathbb{H}$ is said to be precisely invariant under a subgroup $\Gamma_{S}$ of $\Gamma$ if $\gamma(S)=S$ for all $\gamma \in \Gamma_{S}$ and $\gamma(S) \cap S=\emptyset$ for all $\gamma \in \Gamma-\Gamma_{S}$. Furthermore a hyperbolic element $\gamma \in \Gamma$ is said to be simple if the axis of $\gamma$ is precisely invariant under the cyclic subgroup $\langle\gamma\rangle$ generated by $\gamma$. The hyperbolic distance on $\mathbb{H}$ is denoted by $d$. For a quasiconformal automorphism $f$ of $\mathbb{H}$, the maximal dilatation of $f$ is denoted by $K(f)$.

Let $T(\Gamma)$ be the Teichmüller space of $\Gamma$ and $\operatorname{Mod}(\Gamma)$ the Teichmüller modular group of $\Gamma$. It is known that if $\Gamma$ is finitely generated of the first kind, then $T(\Gamma)$ is finite dimensional and the action of $\operatorname{Mod}(\Gamma)$ on $T(\Gamma)$ is properly discontinuous. This means that for every sequence $\left\{f_{n}\right\}_{n=1}^{\infty}$ of quasiconformal automorphisms of $\mathbb{H}$ satisfying $f_{n} \circ \Gamma \circ f_{n}^{-1}=\Gamma$ and $\lim _{n \rightarrow \infty} K\left(f_{n}\right)=1$, there exist an integer $N$ and a conformal automorphism $f$ of $\mathbb{H}$ such that $f_{n}$ are coincident with $f$ on the real axis $\mathbb{R}$ for all $n \geq N$. On the other hand, if $\Gamma$ is infinitely generated, then $T(\Gamma)$ is infinite dimensional and the action of $\operatorname{Mod}(\Gamma)$ is not properly discontinuous, in general. This means that there exists a sequence $\left\{f_{n}\right\}_{n=1}^{\infty}$ of quasiconformal automorphisms $f_{n}$ of $\mathbb{H}$ such that $f_{n} \circ \Gamma \circ f_{n}^{-1}=\Gamma$ and $\lim _{n \rightarrow \infty} K\left(f_{n}\right)=1$. On the basis of this fact, in [3], we proved that if $\Gamma$ satisfies a certain bound condition on translation length, then $\operatorname{Mod}(\Gamma)$ acts properly discontinuously. The following proposition, which gives a lower bound of the maximal dilatation of a quasiconformal automorphism, is crucial for the proof.

Proposition 1 ([3, Proposition 2]). Let $\Gamma$ be a non-elementary Fuchsian group acting on $\mathbb{H}$ and $f$ a quasiconformal automorphism of $\mathbb{H}$ satisfying $f \circ \Gamma \circ f^{-1}=\Gamma$. Suppose that there exist three distinct axes $L_{i}(i=1,2,3)$ of simple hyperbolic elements $\gamma_{i}$ of $\Gamma$ such that $f\left(L_{1}\right)_{*}=L_{1}, f\left(L_{2}\right)_{*}=L_{2}$ and $f\left(L_{3}\right)_{*} \neq L_{3}$, where $f\left(L_{i}\right)_{*}$ is the axis of $f \circ \gamma_{i} \circ f^{-1}$. Let $M$ and $D$ be positive constants such that the translation lengths of $\gamma_{i}(i=1,2,3)$ are less than $M$ 
and $d\left(z_{1}, L_{2}\right) \leq D, d\left(z_{1}, L_{3}\right) \leq D$ for some $z_{1} \in L_{1}$. Then there exists a constant $A=A(M, D)>1$ depending only on $M$ and $D$ such that $K(f) \geq A$.

When $M$ tends to $+\infty$ even with remaining $D$ bounded, the constant $A(M, D)$ might tend to 1 as the following example shows.

Example 2. Let $\Gamma$ be a Fuchsian group such that

$$
R=\mathbb{H} / \Gamma=\mathbb{C}-\bigcup_{n=1}^{\infty} \bigcup_{m \in \mathbb{Z}}\left\{\frac{m}{n}+(2 n+1) \sqrt{-1}\right\}-\{-1,0,1\},
$$

and

$$
\hat{f}_{n}(z)= \begin{cases}x-(y-2 n-2) / n+y \sqrt{-1} & (2 n+1 \leq y<2 n+2) \\ x+(y-2 n) / n+y \sqrt{-1} & (2 n \leq y<2 n+1) \\ x+y \sqrt{-1} & \text { elsewhere }\end{cases}
$$

which are quasiconformal automorphisms of $R$ (see [3, Example 2]). Then $\lim _{n \rightarrow \infty} K\left(\hat{f}_{n}\right)=1$. Let $c_{1}$ be a simple closed geodesic on $R$ surrounding -1 and 0 , and $c_{2}$ a simple closed geodesic on $R$ surrounding 0 and 1 . Furthermore for each $n$, let $c_{3, n}$ be a simple closed geodesic on $R$ surrounding $n+1$ points, 1 and $1+(2 k+1) \sqrt{-1}(k=1, \cdots, n)$. Then the hyperbolic lengths of $c_{3, n}$ tend to $+\infty$ as $n \rightarrow \infty$. Let $L_{i}(i=1,2)$ be an axis of a hyperbolic element $\gamma_{i} \in \Gamma$ such that $\pi\left(L_{i}\right)=c_{i}$. Here $\pi$ is the projection of $\mathbb{H}$ to $R$. We may assume that $L_{1}=\{y \sqrt{-1} \mid y>0\}$. Since $\hat{f}_{n}\left(c_{i}\right)=c_{i}(i=1,2)$ and $c_{1} \cap c_{2} \neq \emptyset$, we can take a lift $f_{n}$ of $\hat{f}_{n}$ to $\mathbb{H}$ so that $f_{n}\left(L_{i}\right)_{*}=L_{i}(i=1,2)$. Furthermore for each $n$, we take an axis $L_{3, n}$ of a primitive hyperbolic element $\gamma_{n} \in \Gamma$ so that $\pi\left(L_{3, n}\right)=c_{3, n}$ and that $d\left(\sqrt{-1}, L_{3, n}\right) \leq \ell_{1}$ for the translation length $\ell_{1}$ of $\gamma_{1}$. Since $\hat{f}_{n}\left(c_{3, n}\right)$ is not homotopic to $c_{3, n}$ for each $n$, we have $f_{n}\left(L_{3, n}\right)_{*} \neq L_{3, n}$. On the other hand, the translation lengths of $\gamma_{n}$ tend to $+\infty$.

On the other hands, when $D$ tends to $+\infty$ even with remaining $M$ bounded, the constant $A(M, D)$ might tend to 1 .

Example 3. Let $\Gamma, R, c_{1}, c_{2}$ and $\hat{f}_{n}$ be the same as in Example 2. For each $n$, let $c_{3, n}$ be a simple closed geodesic on $R$ surrounding two points, $1 / n+(2 n+1) \sqrt{-1}$ and $2 / n+(2 n+1) \sqrt{-1}$. Then the hyperbolic lengths of $c_{3, n}$ are uniformly bounded. Indeed, the lengths are all the same. Let $L_{i}$ $(i=1,2)$ be an axis of a hyperbolic element of $\Gamma$ such that $\pi\left(L_{i}\right)=c_{i}$. We take a lift $f_{n}$ of $\hat{f}_{n}$ to $\mathbb{H}$ so that $f_{n}\left(L_{i}\right)_{*}=L_{i}(i=1,2)$. Let $L_{3, n}$ be an axis of a primitive hyperbolic element $\gamma_{n} \in \Gamma$ so that $\pi\left(L_{3, n}\right)=c_{3, n}$. Since $\hat{f}_{n}\left(c_{3, n}\right)$ is not homotopic to $c_{3, n}$, we have $f_{n}\left(L_{3, n}\right)_{*} \neq L_{3, n}$, and we see that the translation lengths of $\gamma_{n}$ are the same for all $n$. On the other hand, since $d\left(c_{1}, c_{3, n}\right) \rightarrow+\infty$, we have $d\left(L_{1}, L_{3, n}\right) \rightarrow+\infty$. 
In Proposition 1, the invariance of $L_{1}$ and $L_{2}$ under $f$ is used to guarantee that $f$ fixes three points on the boundary. In this case, the condition $d\left(z_{1}, L_{3}\right) \leq D$ implies that the spherical diameter of $L_{3}$ is less than some positive constant $D^{\prime}=D^{\prime}(D)$. Furthermore the assumption on the boundedness of the translation lengths of $\gamma_{i}(i=1,2)$ is used to guarantee that the end points $a$ and $b$ of $L_{3}$ are not in some neighborhoods of the end points of $L_{1}$ and $L_{2}$, and the radii of the neighborhoods are estimated by $M$ and $D$. In particular, we obtained the uniformity of $A$ on $M$ and $D$.

In this paper, we consider a quasiconformal automorphism $f$ of $\mathbb{H}$ fixing three points. We call such a quasiconformal automorphism normalized, and assume that the three points are 0,1 and $\infty$. Suppose that there exist axes $L$ such that $f(L)_{*} \neq L$. Then, each axis $L$ gives a lower bound of the maximal dilatation of $f$, and we will express the lower bound explicitly (Theorem 7). In particular, we can observe the dependence of the lower bound on the translation length, the end points and the Euclidean diameter of $L$. However the uniformity is not taken into account in our theorem.

\section{Results}

Our first theorem gives a lower bound of the Euclidean distance between an end point of an axis and its image under a quasiconformal automorphism.

Theorem 4. Let $\Gamma$ be a torsion-free Fuchsian group acting on $\mathbb{H}$, and $f$ a quasiconformal automorphism of $\mathbb{H}$ such that $f \circ \Gamma \circ f^{-1}=\Gamma$. Furthermore let $L$ be the axis of a simple hyperbolic element $\gamma$ of $\Gamma$ such that $f(L)_{*} \neq L$. Then there exists a positive constant $B$ such that at least one end point a of $L$ satisfies $|f(a)-a| \geq B$, where $B$ depends only on the translation length $\ell$ of $\gamma$, the translation length $\ell^{\prime}$ of $f \circ \gamma \circ f^{-1}$ and the Euclidean diameter $d_{E}(L)$ of L. More precisely,

$$
\begin{gathered}
B=d_{E}(L) \cdot B^{\prime}, \\
B^{\prime}\left(\ell, \ell^{\prime}\right)= \begin{cases}B_{1}^{\prime}\left(\ell, \ell^{\prime}\right):=\frac{1}{2}\left(1-\frac{\sinh (\ell / 2) \sinh \left(\ell^{\prime} / 2\right)}{\cosh (\ell / 2) \cosh \left(\ell^{\prime} / 2\right)+1}\right) & \left(L \cap f(L)_{*}=\emptyset\right) \\
B_{2}^{\prime}\left(\ell, \ell^{\prime}\right):=\frac{\delta}{1-\delta} & \left(L \cap f(L)_{*} \neq \emptyset\right),\end{cases} \\
\delta=\delta\left(\ell, \ell^{\prime}\right)=\frac{1}{12}\left(1-\sqrt{\left.1-\frac{1}{\sinh ^{2}(\ell / 2) \sinh ^{2}\left(\ell^{\prime} / 2\right)}\right) .}\right.
\end{gathered}
$$

Remark. The constant $B_{1}^{\prime}\left(\ell, \ell^{\prime}\right)$ tends to $1 / 2$ as $\ell, \ell^{\prime} \rightarrow 0$ and tends to 0 as $\ell, \ell^{\prime} \rightarrow+\infty$.

A proof of Theorem 4 is given in Section 4. In this section, assuming this result, we give a lower bound of the maximal dilatations of the quasiconformal homeomorphisms. The following two lemmas tell us a relationship between 
the Euclidean distance which we considered in Theorem 4 and the maximal dilatation. The first one is due to Teichmüller (see [5]).

Lemma 5. Let $f$ be a quasiconformal automorphism of $\mathbb{C}$ fixing 0 and 1 . Suppose that there exists a point $z_{0} \in \mathbb{C}-\{0,1\}$ such that $d_{0,1}\left(z_{0}, f\left(z_{0}\right)\right)=\log C$ for some constant $C>1$, where $d_{0,1}$ is the hyperbolic distance on the twice punctures plane $\mathbb{C}-\{0,1\}$. Then $K(f) \geq C^{2}$.

If $z_{0}, f\left(z_{0}\right)<0$, then the exact computation of $d_{0,1}\left(z_{0}, f\left(z_{0}\right)\right)$ is given by Sugawa and Vuorinen [7, Lemma 5.1].

Lemma 6. The hyperbolic distance between $-x$ and $-y$ in $\mathbb{C}-\{0,1\}$ is given by $d_{0,1}(-x,-y)=|\Phi(x)-\Phi(y)|$ for $x, y>0$. The function $\Phi: \mathbb{R} \rightarrow \mathbb{R}$ is given by

where

$$
\Phi(x)=\frac{1}{2} \log \frac{\mathcal{K}(x /(1+x))}{\mathcal{K}(1 /(1+x))}
$$

$$
\mathcal{K}(z)=\int_{0}^{1} \frac{d t}{\sqrt{\left(1-t^{2}\right)\left(1-z t^{2}\right)}} .
$$

Now we state our second theorem, which easily follows from Theorem 4 with Lemmas 5 and 6 .

Theorem 7. Let $\Gamma$ be a torsion-free Fuchsian group acting on $\mathbb{H}$ and $f$ a normalized quasiconformal automorphism of $\mathbb{H}$ such that $f \circ \Gamma \circ f^{-1}=\Gamma$. Suppose that there exists an axis $L$ of a simple hyperbolic element $\gamma$ of $\Gamma$ such that $f(L)_{*} \neq L$. Then there exists a constant $A>1$ such that $K(f) \geq A$, where $A$ depends only on the translation length $\ell$ of $\gamma$, the translation length $\ell^{\prime}$ of $f \circ \gamma \circ f^{-1}$ and the end points $a$ and $b$ of $L$. More precisely, under the assumption that $|f(b)-b| \leq|f(a)-a|$ and $a<0$,

$$
\frac{1}{2} \log A= \begin{cases}\Phi(-a+B)-\Phi(-a) & (f(a)<a) \\ \Phi(-a)-\Phi(-a-B) & (f(a)>a) .\end{cases}
$$

The constant $B$ and the function $\Phi$ are the same as in Theorem 4 and Lemma 6 .

Proof. Since $a<0$ and $f$ is normalized, we have $f(a)<0$. By Theorem 4 , the end point $a$ satisfies $|f(a)-a| \geq B$. Furthermore, by Lemma 6 , the hyperbolic distance between $f(a)$ and $a$ in $\mathbb{C}-\{0,1\}$ is given by $d_{0,1}(f(a), a)=$ $|\Phi(-f(a))-\Phi(-a)|$. Since the function $\Phi(x)$ is strictly increasing for $x>0$, we see that $d_{0,1}(f(a), a) \geq \Phi(-a+B)-\Phi(-a)$ if $f(a)<a$ and $d_{0,1}(f(a), a) \geq$ $\Phi(-a)-\Phi(-a-B)$ if $f(a)>a$. Hence Lemma 5 yields the assertion.

Remark. (i) In Theorem 7, the assumption that $a<0$ is not essential. Also in the case where $0<a<1$ or $1<a$, we have similar results by using the 
fact that $d_{0,1}\left(z_{1}, z_{2}\right) \geq\left|\Phi\left(\left|z_{1}\right|\right)-\Phi\left(\left|z_{2}\right|\right)\right|$ for $z_{1}, z_{2} \in \mathbb{C}-\{0,1\}$ (see [7, Lemma 5.16]). (ii) For a Fuchsian group $\Gamma$ and a quasiconformal automorphism $f$ of $\mathbb{H}$ satisfying $f \circ \Gamma \circ f^{-1}=\Gamma$, we have $K(f) \geq \max \left\{\ell / \ell^{\prime}, \ell^{\prime} / \ell\right\}$, where $\ell$ is the translation length of an element $\gamma \in \Gamma$ and $\ell^{\prime}$ is the translation length of $f \circ \gamma \circ f^{-1}$ (see [8, Lemma 3.1]). This is trivial if $\ell=\ell^{\prime}$. However our theorem gives a non-trivial estimate also for the case where $\ell=\ell^{\prime}$. Indeed, in Theorem 4, we have

$$
B_{1}^{\prime}(\ell, \ell)=\frac{2}{\cosh \ell+3}
$$

and

$$
\delta(\ell, \ell)=\frac{1}{12}\left(1-\sqrt{1-\left(\frac{2}{\cosh \ell-1}\right)^{2}}\right) .
$$

(iii) In Proposition 1, the constant $A$ does not depend on the translation lengths $\ell_{i}^{\prime}$ of $f \circ \gamma_{i} \circ f^{-1}(i=1,2,3)$. Indeed, in the proof of the proposition, we assume that $K(f)<2$ and use the fact that $\ell_{i}^{\prime}<2 \ell_{i}$, where $\ell_{i}$ is the translation length of $\gamma_{i}$. Also in our theorem, if we assume that $\ell^{\prime}<2 \ell$, then we obtain a constant $A=A(\ell, a, b)$ with $B^{\prime}=B^{\prime}(\ell, 2 \ell)$.

When we can take $a=-1$ in Theorem 7 , we express a lower bound of the maximal dilatation simply.

Corollary 8. Let $\Gamma$ be a torsion-free Fuchsian group acting on $\mathbb{H}$, and $f$ a normalized quasiconformal automorphism of $\mathbb{H}$ such that $f \circ \Gamma \circ f^{-1}=\Gamma$. Suppose that there exists a simple hyperbolic element $\gamma \in \Gamma$ such that one end point of the axis $L$ of $\gamma$ is -1 and that $f(-1)<-1$ and that $|f(b)-b| \leq$ $|f(-1)+1|$, where $b$ is another end point of $\gamma$. Then

$$
K(f) \geq 1+\frac{\log (1+B)}{E},
$$

where $B$ is the same constant as in Theorem 4 and

$$
E=\frac{4}{\pi} \mathcal{K}\left(\frac{1}{2}\right)^{2} \approx 4.37688 .
$$

Proof. Since $\Phi(1)=0$, Theorem 7 yields that $K(f) \geq A$, where $(1 / 2) \log A=$ $\Phi(1+B)$. By $[7$, Lemma 5.4], we have

$$
\Phi(1+B) \geq \frac{1}{2} \log \left(1+\frac{\log (1+B)}{E}\right),
$$

which implies the assertion. 


\section{Collar lemma and its application}

This section is devoted to preliminaries for a proof of Theorem 4. The following lemma is known as the collar lemma (see [2], [4] and [6]).

Lemma 9. Let $\Gamma$ be a torsion-free Fuchsian group acting on $\mathbb{H}$, and $L$ the axis of a simple hyperbolic element $\gamma \in \Gamma$ with translation length $\ell>0$. Then a collar

$$
C(L, \omega(\ell))=\{z \in \mathbb{H} \mid d(z, L) \leq \omega(\ell)\}
$$

of $L$ with width $\omega(\ell)$ is precisely invariant under the cyclic subgroup $\langle\gamma\rangle$ generated by $\gamma$, where

$$
\omega(\ell)=\operatorname{arcsinh}\left(\frac{1}{\sinh (\ell / 2)}\right) .
$$

Furthermore, $C(L, \omega(\ell)) \cap C\left(L^{\prime}, \omega\left(\ell^{\prime}\right)\right)=\emptyset$ for every pair of two disjoint axes $L$ and $L^{\prime}$ of simple hyperbolic elements of $\Gamma$ with translation lengths $\ell$ and $\ell^{\prime}$ respectively.

Lemma 9 immediately yields the following.

Corollary 10. Let $\Gamma$ be a torsion-free Fuchsian group acting on $\mathbb{H}$, and $L$ and $L^{\prime}$ two disjoint axes of simple hyperbolic elements of $\Gamma$ with translation lengths $\ell$ and $\ell^{\prime}$ respectively. Then the hyperbolic distance $d\left(L, L^{\prime}\right)$ satisfies

$$
d\left(L, L^{\prime}\right) \geq \operatorname{arccosh}\left(\frac{\cosh (\ell / 2) \cosh \left(\ell^{\prime} / 2\right)+1}{\sinh (\ell / 2) \sinh \left(\ell^{\prime} / 2\right)}\right) .
$$

Proof. By Lemma 9, we have $d\left(L, L^{\prime}\right) \geq \omega(\ell)+\omega\left(\ell^{\prime}\right)$. The assertion follows from the following computation:

$$
\begin{aligned}
\cosh \left(\omega(\ell)+\omega\left(\ell^{\prime}\right)\right) & \\
= & \cosh (\omega(\ell)) \cosh \left(\omega\left(\ell^{\prime}\right)\right)+\sinh (\omega(\ell)) \sinh \left(\omega\left(\ell^{\prime}\right)\right) \\
= & \cosh \left\{\operatorname{arcsinh}\left(\frac{1}{\sinh (\ell / 2)}\right)\right\} \cosh \left\{\operatorname{arcsinh}\left(\frac{1}{\sinh \left(\ell^{\prime} / 2\right)}\right)\right\} \\
& +\sinh \left\{\operatorname{arcsinh}\left(\frac{1}{\sinh (\ell / 2)}\right)\right\} \sinh \left\{\operatorname{arcsinh}\left(\frac{1}{\sinh \left(\ell^{\prime} / 2\right)}\right)\right\} \\
= & \left\{\left(\frac{1}{\sinh ^{2}(\ell / 2)}+1\right)\left(\frac{1}{\sinh ^{2}\left(\ell^{\prime} / 2\right)}+1\right)\right\}^{1 / 2}+\frac{1}{\sinh (\ell / 2) \sinh \left(\ell^{\prime} / 2\right)} \\
= & \frac{\cosh (\ell / 2) \cosh \left(\ell^{\prime} / 2\right)+1}{\sinh (\ell / 2) \sinh \left(\ell^{\prime} / 2\right)} .
\end{aligned}
$$

Next, for two axes which intersect each other, we will obtain a lower bound of the intersection angle. Note that for two axes $L$ and $L^{\prime}$ of simple hyperbolic 
elements $\gamma$ and $\gamma^{\prime}$ of $\Gamma$ such that $L \cap L^{\prime} \neq \emptyset$, the translation lengths $\ell$ and $\ell^{\prime}$ of $\gamma$ and $\gamma^{\prime}$ respectively satisfy

$$
\sinh \left(\frac{\ell}{2}\right) \sinh \left(\frac{\ell^{\prime}}{2}\right)>1
$$

Indeed, since $L^{\prime}$ passes the collar of $L$ with width $\omega(\ell)$, we have $\ell^{\prime}>2 \omega(\ell)$.

Proposition 11. Let $\Gamma$ be a torsion-free Fuchsian group acting on $\mathbb{H}$, and $L$ and $L^{\prime}$ two distinct axes of simple hyperbolic elements $\gamma$ and $\gamma^{\prime}$ of $\Gamma$ with translation lengths $\ell$ and $\ell^{\prime}$ respectively. Suppose that $L \cap L^{\prime} \neq \emptyset$. Then the intersection angle $\theta\left(L, L^{\prime}\right)$ between $L$ and $L^{\prime}$ satisfies

$$
\theta\left(L, L^{\prime}\right) \geq \arcsin \left(\frac{1}{\sinh (\ell / 2) \sinh \left(\ell^{\prime} / 2\right)}\right) .
$$

Proof. By Lemma 9, we can take a collar $C(L, \omega(\ell))$ of $L$ with width

$$
\omega(\ell)=\operatorname{arcsinh}\left(\frac{1}{\sinh (\ell / 2)}\right) .
$$

Then, by the formula [1, Theorem 7.11.2] for right-angled triangles, the length $\ell_{0}$ of the segment $L^{\prime} \cap C(L, \omega(\ell))$ satisfies

$$
\sinh \omega(\ell)=\sinh \left(\ell_{0} / 2\right) \cdot \sin \left(\theta\left(L, L^{\prime}\right)\right)
$$

Since the translation length of $\gamma^{\prime}$ is $\ell^{\prime}$, it is clear that $\ell_{0} \leq \ell^{\prime}$. Then we see that

$$
\sin \left(\theta\left(L, L^{\prime}\right)\right)=\frac{\sinh \omega(\ell)}{\sinh \left(\ell_{0} / 2\right)} \geq \frac{1}{\sinh (\ell / 2) \sinh \left(\ell^{\prime} / 2\right)} .
$$

In the last of this section, we mention the hyperbolic distance and the angle between an axis and its image under a conformal automorphism.

Corollary 12. Let $\Gamma$ be a torsion-free Fuchsian group acting on $\mathbb{H}, L$ the axis of a simple hyperbolic element of $\Gamma$ with translation length $\ell$, and $f$ a conformal automorphism of $\mathbb{H}$ satisfying $f \circ \Gamma \circ f^{-1}=\Gamma$. Suppose that $f(L) \neq L$. If $f(L) \cap L=\emptyset$, then

$$
d(f(L), L) \geq \operatorname{arccosh}\left(\frac{\cosh \ell+3}{\cosh \ell-1}\right) .
$$

If $f(L) \cap L \neq \emptyset$, then the intersection angle $\theta(f(L), L)$ between $f(L)$ and $L$ satisfies

$$
\theta(f(L), L) \geq \arcsin \left(\frac{2}{\cosh \ell-1}\right) .
$$

Proof. We may put $\ell=\ell^{\prime}$ in Corollary 10 and Proposition 11. 


\section{Proof of Theorem 4}

For a proof of Theorem 4, first we prove the following.

Lemma 13. Let $\Gamma$ be a torsion-free Fuchsian group acting on $\mathbb{H}$ and $L$ and $L^{\prime}$ two distinct axes of simple hyperbolic elements of $\Gamma$ with translation lengths $\ell$ and $\ell^{\prime}$ respectively. Suppose that $L=\{i y \mid y>0\}$. Then the two end points $x$ of $L^{\prime}$ satisfy $|x| \geq \delta^{\prime}$, where

$$
\delta^{\prime}= \begin{cases}\frac{d_{E}\left(L^{\prime}\right)}{2}\left(\frac{\cosh (\ell / 2) \cosh \left(\ell^{\prime} / 2\right)+1}{\sinh (\ell / 2) \sinh \left(\ell^{\prime} / 2\right)}-1\right) & \left(L \cap L^{\prime}=\emptyset\right) \\ \frac{d_{E}\left(L^{\prime}\right)}{2}\left(1-\sqrt{1-\frac{1}{\sinh ^{2}(\ell / 2) \sinh ^{2}\left(\ell^{\prime} / 2\right)}}\right) & \left(L \cap L^{\prime} \neq \emptyset\right) .\end{cases}
$$

Proof. First we suppose that $L \cap L^{\prime}=\emptyset$. Without loss of generality, we may assume that the two end points $x_{1}$ and $x_{2}$ of $L^{\prime}$ satisfy $0<x_{1}<x_{2}$. By Corollary 10, we have

$$
d\left(L, L^{\prime}\right) \geq \operatorname{arccosh}\left(\frac{\cosh (\ell / 2) \cosh \left(\ell^{\prime} / 2\right)+1}{\sinh (\ell / 2) \sinh \left(\ell^{\prime} / 2\right)}\right):=D\left(\ell, \ell^{\prime}\right) .
$$

Let $L_{0}$ be a geodesic on $\mathbb{H}$ such that $d\left(L, L_{0}\right)=D\left(\ell, \ell^{\prime}\right)$ and $d_{E}\left(L_{0}\right)=d_{E}\left(L^{\prime}\right)$, and $x_{1}^{0}$ and $x_{2}^{0}$ the end points of $L_{0}$. We may assume that $0<x_{1}^{0}<x_{2}^{0}$. Then $x_{1} \geq x_{1}^{0}$. Let $\theta_{0}$ be an angle such that $\cos \theta_{0}=\left\{\cosh \left(D\left(\ell, \ell^{\prime}\right)\right)\right\}^{-1}$. Then we see that

$$
\left(x_{1}^{0}+\frac{d_{E}\left(L^{\prime}\right)}{2}\right) \cos \theta_{0}=\frac{d_{E}\left(L^{\prime}\right)}{2}
$$

(see $[1,(7.20 .3)])$. Thus we have

$$
\begin{aligned}
x_{1}^{0} & =\frac{d_{E}\left(L^{\prime}\right)}{2} \frac{1-\cos \theta_{0}}{\cos \theta_{0}}=\frac{d_{E}\left(L^{\prime}\right)}{2}\left\{\cosh \left(D\left(\ell, \ell^{\prime}\right)\right)-1\right\} \\
& \geq \frac{d_{E}\left(L^{\prime}\right)}{2}\left(\frac{\cosh (\ell / 2) \cosh \left(\ell^{\prime} / 2\right)+1}{\sinh (\ell / 2) \sinh \left(\ell^{\prime} / 2\right)}-1\right) .
\end{aligned}
$$

Next we suppose that $L \cap L^{\prime} \neq \emptyset$, and let $\theta$ be the intersection angle. Then by Proposition 11, we have

$$
\cos \theta \leq \cos \left\{\arcsin \left(\frac{1}{\sinh (\ell / 2) \sinh \left(\ell^{\prime} / 2\right)}\right)\right\}=\sqrt{1-\frac{1}{\sinh ^{2}(\ell / 2) \sinh ^{2}\left(\ell^{\prime} / 2\right)}} .
$$

Without loss of generality, we may assume that the two end points $x_{1}$ and $x_{2}$ of $L^{\prime}$ satisfy $x_{1}<0<x_{2}$ and $\left|x_{1}\right| \leq\left|x_{2}\right|$. Then $x_{1}+d_{E}\left(L^{\prime}\right) / 2 \geq 0$ and

$$
\left|x_{1}\right|=\frac{d_{E}\left(L^{\prime}\right)}{2}(1-\cos \theta) \geq \frac{d_{E}\left(L^{\prime}\right)}{2}\left(1-\sqrt{1-\frac{1}{\sinh ^{2}(\ell / 2) \sinh ^{2}\left(\ell^{\prime} / 2\right)}}\right) .
$$


Now we prove our theorem.

Proof of Theorem 4. We may assume that neither $a$ nor $f(a)$ is $\infty$. First we suppose that $f(L)_{*} \cap L=\emptyset$. Let $\Omega$ be a simply connected domain that is surrounded by $L, f(L)_{*}$ and a subset of $\mathbb{R}$. If $\Omega$ is not bounded, then it is clear that at least one end point $a$ satisfies

$$
|f(a)-a|>d_{E}(L) .
$$

Thus we have only to consider the case where $\Omega$ is bounded. By translation along the real axis, we may assume that $L$ is symmetrical about the imaginary axis, and then passes through $\left(d_{E}(L) / 2\right) i$. By Corollary 10, we have

$$
d\left(f(L)_{*}, L\right) \geq \operatorname{arccosh}\left(\frac{\cosh (\ell / 2) \cosh \left(\ell^{\prime} / 2\right)+1}{\sinh (\ell / 2) \sinh \left(\ell^{\prime} / 2\right)}\right)=: d_{0} .
$$

Let $L_{0}$ be a geodesic on $\mathbb{H}$ such that it is symmetrical about the imaginary axis and satisfies $d\left(L_{0}, L\right)=d_{0}$ and passes through $\left(d_{E}(L) / 2\right) \cdot e^{-d_{0}} i$. Then the end point $c>0$ of $L$ and the end point $c_{0}>0$ of $L_{0}$ satisfy $\left|c-c_{0}\right|=$ $\left(d_{E}(L) / 2\right) \cdot\left(1-e^{-d_{0}}\right)=: d_{1}$. It is easily seen that at least one end point $a$ of $f(L)_{*}$ satisfies

$$
\begin{aligned}
|f(a)-a| & \geq d_{1} \\
& \geq \frac{d_{E}(L)}{2}\left(1-\exp \left\{-\operatorname{arccosh}\left(\frac{\cosh (\ell / 2) \cosh \left(\ell^{\prime} / 2\right)+1}{\sinh (\ell / 2) \sinh \left(\ell^{\prime} / 2\right)}\right)\right\}\right) \\
& \geq \frac{d_{E}(L)}{2}\left(1-\frac{\sinh (\ell / 2) \sinh \left(\ell^{\prime} / 2\right)}{\cosh (\ell / 2) \cosh \left(\ell^{\prime} / 2\right)+1}\right),
\end{aligned}
$$

and we have the assertion.

Next we suppose that $f(L)_{*} \cap L \neq \emptyset$. Furthermore we may assume that the Euclidean diameter $d_{E}\left(f(L)_{*}\right)$ of $f(L)_{*}$ satisfies $2 d_{E}(L)>d_{E}\left(f(L)_{*}\right)>$ $d_{E}(L) / 2$. Indeed, if $d_{E}\left(f(L)_{*}\right) \leq d_{E}(L) / 2$, then at least one end point $a$ of $L$ satisfies

$$
|f(a)-a|>d_{E}(L) / 2 .
$$

If $2 d_{E}(L) \leq d_{E}\left(f(L)_{*}\right)$, then at least one end point $a$ of $L$ satisfies

$$
|f(a)-a|>d_{E}(L) .
$$

Since $\delta /(1-\delta)<1 / 2$, in these cases, we have the assertion.

Let $\alpha$ and $\beta$ be the two end points of $L$, and $\alpha^{\prime}$ and $\beta^{\prime}$ be the two end points of $f(L)_{*}$. We may assume that $\alpha^{\prime}<\alpha<\beta^{\prime}<\beta$. We have only to prove that $\alpha-\alpha^{\prime}>B$ under the assumption that $f(\alpha)=\alpha^{\prime}$. Indeed, if $f(\beta)=\alpha^{\prime}$, then $\beta-f(\beta)=\beta-\alpha^{\prime}>\alpha-\alpha^{\prime}>B$. We take a Möbius transformation $\phi(z)=(z-\alpha) /(z-\beta)$. Then $\phi(L)=\{y \sqrt{-1} \mid y>0\}$. Furthermore

$$
d_{E}\left(\phi\left(f(L)_{*}\right)\right)=\left|\phi\left(\alpha^{\prime}\right)-\phi\left(\beta^{\prime}\right)\right|=\frac{(\beta-\alpha)\left(\beta^{\prime}-\alpha^{\prime}\right)}{\left(\beta-\alpha^{\prime}\right)\left(\beta-\beta^{\prime}\right)} .
$$


Here we note that

$$
\beta-\alpha^{\prime}<\left(\beta^{\prime}-\alpha^{\prime}\right)+(\beta-\alpha)=d_{E}\left(f(L)_{*}\right)+d_{E}(L)<3 d_{E}(L),
$$

and

$$
\beta-\beta^{\prime}<\beta-\alpha=d_{E}(L)
$$

Thus

$$
d_{E}\left(\phi\left(f(L)_{*}\right)\right)>\frac{d_{E}(L) \cdot\left(d_{E}(L) / 2\right)}{3 d_{E}(L) \cdot d_{E}(L)}=\frac{1}{6} .
$$

By Lemma 13, we see that $\left|\phi\left(\alpha^{\prime}\right)\right|>\delta$. Since $\left|\phi\left(\alpha^{\prime}\right)\right|=\left(\alpha-\alpha^{\prime}\right) /\left(\beta-\alpha^{\prime}\right)$, we have

$$
\alpha-\alpha^{\prime}>\delta\left(\beta-\alpha^{\prime}\right)=\delta\left(\left(\alpha-\alpha^{\prime}\right)+(\beta-\alpha)\right) .
$$

Since $\beta-\alpha=d_{E}(L)$ and $\delta<1 / 12$, the above inequality is equivalent to

$$
\alpha-\alpha^{\prime}>\frac{\delta}{1-\delta} \cdot d_{E}(L) \text {. }
$$

\section{REFERENCES}

[1] A. F. Beardon, The Geometry of Discrete Groups, Graduate Texts in Mathematics 91, Springer, 1983.

[2] P. Buser, Geometry and Spectra of Compact Riemann Surfaces, Progress in Mathematics 106, Birkhäuser Boston-Basel-Berlin, 1992.

[3] E. Fujikawa, H. Shiga and M. Taniguchi, On the action of the mapping class group for Riemann surfaces of infinite type, J. Math. Soc. Japan 56 (2004), to appear.

[4] N. Halpern, A proof of the collar lemma, Bull. London Math. Soc. 13 (1981), 141-144.

[5] I. Kra, On Teichmüller's theorem on the quasi-invariance of cross ratios, Israel. J. Math. 30 (1978), 152-158

[6] J. P. Matelski, A compactness theorem for Fuchsian groups of the second kind, Duke Math. J. 43 (1976), 829-840.

[7] T. Sugawa and M. Vuorinen, Some inequalities for the Poincaré metric of plane domain, preprint.

[8] S. A. Wolpert, The length spectra as moduli for compact Riemann surfaces, Ann. of Math. 109 (1979), 323-351.

Research Institute for Mathematical Sciences, Kyoto University, Kyoto, 606-8502, JAPAN

Current address: Department of Mathematical and Computing Sciences, Tokyo Institute of Technology, Oh-okayama Meguro-ku Tokyo, 152-8552, Japan

E-mail address: ege.fujikawa@is.titech.ac.jp 